\title{
The Pattern of Parasite Density, Plasma Total Bile Acids and Lactate Dehydrogenase in Plasmodium Infected Patients in Rural Community
}

\author{
Mathew Folaranmi OLANIYAN*, Elizabeth Moyinoluwa BABATUNDE \\ Department of Medical Laboratory Science, Achievers University, Owo, Ondo state -Nigeria \\ *Corresponding author: olaniyanmat@yahoo.com
}

Received June 07, 2014; Revised June 26, 2014; Accepted July 09, 2014

\begin{abstract}
Background to the study: Pathophysiology of Plasmodium (vivax, ovale, falciparum and malariae) infection involves liver. Liver dysfunction and destruction of tissues could be indicated by the plasma level of Total Bile Acid (TBA) and Lactate Dehydrogenase (LDH). Aim and Objective: This work was designed to evaluate the pattern of parasite density, plasma total bile acids, and Lactate dehydrogenase in Plasmodium infected patients in rural community. Materials and Methods: The study was carried out in Kishi, the Headquarter of Irepo Local government area of Oyo state - Nigeria. Seven hundred and nine (709) subjects (Female: $n=403$ : male: $n=306$ ) aged 5 to 68 years were tested for Plasmodium infection using Giemsa- thick film technique. The overall prevalence of Plasmodium infection among the seven hundred and nine subjects screened was found to be $29.1 \%$ (206) including 12.97\% (92) HIV, HBsAg and anti-HCV seronagative patients and 16.1\% (114) HIV, HBsAg or anti-HCV seropositive patients. Ninety two $(92(12.97 \%))$ that were HIV, HBsAg and anti-HCV seronagative(female-58 (63.0\%); male-34 (37\%)) were recruited out of 206 (29.1\%) that were found to be infected with plasmodium spp for the study. None of the subject was jaundiced as at the time of sample collection. Hepatitis B surface antigen (HBsAg) and anti-HCV tests were carried out by Enzyme Linked Immunozorbent Assay (ELIZA). HIV screening and confirmation were carried out by immuno-chromatographic and Immunobloting (Western blot) assays respectively. Fasting Plasma Total Bile Acids (TBA) and Lactate Dehydrogenase (LDH) were analyzed in the patients biochemically by spectrophotometry. Result: The result obtained showed an overall prevalence of Plasmodium infection as 29.1\% (206) (female: 107 (52\%); male: 99 (48\%)) including 12.97\% (92) (Female: 58 (63.0\%) Male: 34 (37\%)) that were HIV, HBsAg and anti-HCV seronagative and 16.1\% (114) (Female: 61 (53.5\%); Male: 53 (46.5\%)) were co-infected with at least one of HIV, HBV or HCV. The plasmodium infected subjects were grouped into three based on the parasite density such as: patients with parasite density of 50-499; 500-999 and $\geq 1000$. The mean value of the parasite density of each group was correlated with the plasma level of LDH and TBA. In all groups there was a strong positive correlation $(\mathrm{R}=1 ; \mathrm{R} 2=1)$ between the plasma TBA, $\mathrm{LDH}$ and plasmodium parasite density. The pattern of parasite density obtained in the rural community studied include $45.7 \%$ had a mean parasite density of $282 \pm 12.0 ; 43.5 \%(853 \pm 31.0)$ and $10.9 \%(1130 \pm 61.0)$. There was also a statistical significant increase in the mean value of LDH and TBA with increase in parasite density with $\mathrm{p}<0.05$. Conclusion: This work showed an overall prevalence of $29.1 \%$ (206) plasmodium infection including $16.1 \%$ (114) of the patients co-infected with at least one of HIV, HBV or HCV. The plasma level of LDH and TBA was also found to be positively correlated and directly proportional to the parasite density. Evaluation of these parameters is therefore recommended for effective control and management.
\end{abstract}

Keywords: prevalence, LDH, TBA, parasite density, plasmodium, correlation

Cite This Article: Mathew Folaranmi OLANIYAN, and Elizabeth Moyinoluwa BABATUNDE, "The Pattern of Parasite Density, Plasma Total Bile Acids and Lactate Dehydrogenase in Plasmodium Infected Patients in Rural Community.” American Journal of Biomedical Research, vol. 2, no. 3 (2014): 47-51. doi: 10.12691/ajbr-23-2.

\section{Introduction}

The species of Plasmodium (P) that cause malaria in human which include: $P$. vivax. $P$. ovale, $P$. malariae and $P$. falciparum are transmitted by female anopheles mosquito when the mosquito is taking a blood meal from man. The pre-erythrocytic schizogony takes place in the liver. The presentation of malaria may include headache, fever, shivering, joint pain, vomiting, hemolytic anemia, jaundice, hemoglobin in the urine, retinal damage, and convulsions [1].

Lactate dehydrogenase (LDH) catalyzes the interconversion of lactic acid and pyruvic acid. The enzyme is composed of 4 peptide chains and exists in 5 
isomeric forms. $\mathrm{LDH}$ is widely distributed throughout the body; highest concentrations are found in the liver, heart and skeletal muscle. $\mathrm{LDH}$ is a general marker of tissue damage and is often used to determine the root cause and location of damage. LD activity is significantly elevated during myocardial infarction. Maximum levels are reached 24 to 48 hours after the onset of chest pain and may remain elevated for 7 to 12 days postinfarction. Increased activity is also associated with stroke, kidney disease, liver disease, progressive muscular dystrophy, cancer, intestinal and pulmonary infarction [2].

Bile acids are synthesised in the liver as a breakdown product of cholesterol and secreted into the gall bladder. They are released into the small intestine where they solubilise dietary lipids such as cholesterol, aiding their absorption. Bile acids are reabsorbed from the portal blood by hepatocyte extraction and re-excreted into bile, passing through the enterohepatic circulation several times before final excretion [3]. The measurement of Total bile acids (TBA) in serum is a sensitive indicator of liver function. Fasting serum bile acids can be used in the diagnosis and prognosis of liver disease. Levels rise in many liver diseases, for example hepatitis and liver sclerosis. Abnormal levels in fasting patients or immediately after a meal can be used to detect liver disease and damage, impaired liver function, intestinal dysfunction and perhaps a gall bladder blockage [4,5]. Bile acid measurement may detect some forms of liver disease earlier than standard liver tests because bile acids levels correspond to liver function, rather than liver damage. In veterinary medicine, bile acid measurement is considered to be a superior indicator of liver disease. Bile Acids provides a highly specific marker for use in the diagnosis and monitoring of various liver conditions supplying information that conventional markers like AST and ALT cannot. It is also one of the most sensitive markers for the early detection of liver dysfunction. Abnormal levels of Bile Acids are associated with obstetric cholestasis in pregnant women, hepatitis, liver sclerosis and liver cancer [6].

This work is therefore designed to evaluate The pattern of Parasite density, plasma total bile acids and Lactate dehydrogenase in Plasmodium infected patients in a rural community.

\section{Materials and Methods}

\subsection{Materials}

\subsubsection{Study Area}

The study was carried out in Kishi which is the Headquarter of Irepo Local government area of Oyo state - Nigeria. It shares border with Kaima local government area in Kwara state-Nigeria, Oorelope, Olorunsogo local government areas at the northern part of Oyo state, Nigeria and the Republic of Benin.

\subsubsection{Study Population}

Seven hundred and nine (709) were screened for Plasmodium infection classified into female $(n=403)$; male: $(n=306)$ aged 5 to 68 years. The overall prevalence of Plasmodium infection among the seven hundred and nine screened was found to be $29.1 \%(206)$ including 12.97\% (92) HIV, HBsAg and anti-HCV seronagative patients and $16.1 \%$ (114) HIV, HBsAg or anti-HCV seropositive patients. Ninety two(92(12.97\%)) that were HIV, HBsAg and anti-HCV seronagative (female-58 (63.0\%); male-34 (37\%)) were recruited out of 206 (29.1\%) that were found to be infected with plasmodium spp for the study. None of the subject was jaundiced as at the time of sample collection.

\subsubsection{Sample Size}

Ninety two (92 (12.97\%)) that were HIV, HBsAg and anti-HCV seronagative (female-58 (63.0\%); male-34 (37\%)) were recruited out of 206 (29.1\%) that were found to be infected with plasmodium spp for the study based on the exclusion and inclusion criteria.

\subsection{Case Selection Procedure/s}

\subsubsection{Inclusion Criteria}

Anicteric Plasmodium infected patients that were HIV, HBsAg and anti-HCV seronagative aged 5 -68 years were included in the study.

\subsubsection{Exclusion Criteria}

1. Plasmodium infected patients that were HIV, HBsAg and anti-HCV seropositive were excluded.

2. Icteric Plasmodium infected HIV, HBsAg and anti$\mathrm{HCV}$ seronagative patients aged 5 - 68 years were not recruited for the study.

3. Anicteric Plasmodium infected HIV, HBsAg and anti-HCV seropositive patients aged 5 -68 years were not included in the study.

\subsubsection{Blood Sample}

Five (5) milliliter of blood was collected into lithium heparinized bottle from each of the test subjects after an overnight fasting for the estimation of Plasmodium parasite density, LDH, HIV, HBsAg and anti-HCV tests, and Total Bile Acids.

\subsection{Methods}

a. Estimation of Total Bile Acids was carried out on the plasma samples of the subjects using Randox reagent kit. The manufacturer's instruction was strictly followed.

Principle: Two reactions are combined in this kinetic enzyme cycling method. In the first reaction bile acids are oxidised by $3-\alpha$ hydroxysteroid dehydrogenase with the subsequent reduction of Thio-NAD to Thio-NADH. In the second reaction the oxidised bile acids are reduced by the same enzyme with the subsequent oxidation of NADH to NAD. The rate of formation of Thio-NADH is determined by measuring the specific absorbance change at $405 \mathrm{~nm}$.

(Abreviations: NADH, NAD, Thio-NADH, Thio-NAD).

b. Screening for HIV Antibodies HIV screening were carried out using Immuno chromatographic kit (Chembio HIV 1 and 2 STAT-PAK). Positive samples were further confirmed by Western blot/Immunoblotting using ImmunoeticsQualicode TM HIV 1 and 2 kit.

c. Screening for HbsAg by Enzyme- Linked Immunosorbent Assay (ELISA) The ELISA kit from BIORAD Monolisa HBsAg ULTRA EIA92430 Marnes- 
La-Coqutte- France was used. ELISA was done according to the manufactures instruction. The Optical density OD was read at 450/620 to 700 nanometre. The cut off value was determined by the mean of negative control +0.05 (0.08). The test is valid if all values of negative control are lower or equal to 0.08 and Positive control was over 0.08 or equal to 1.0. A test sample is considered negative if the ratio value of sample: cut off value is lower than 1.0 and positive if equal to or greater than 1.0.

d. Screening for HCV Antibody by ELISA ELISA kit from DIA PRO Diagnostic Bioprobes 20099 Sesto San Giovanni (Milano)-Italy was used. ELISA was done according to the manufactures instruction The Optical density OD is read at 450/620 to 700 nanometre. The cut off value is calculated as follows: NC (negative control) $+350=$ cut-off $(\mathrm{C})$, Calibrator mean value $=0.540, \mathrm{~S} / \mathrm{C}=1.4$ (where $\mathrm{S}=$ sample and $\mathrm{C}$ - cut off). $\mathrm{S} / \mathrm{C}=$ higher than 1.1. Any sample with a ratio value of sample /cut off less than 0.9 was considered negative and if higher than 1.1 is positive.

e. Plasma Lactate Dehydrogenase was estimated in the subjects using reagent kit of Randox. The manufacturer's instruction was followed strictly.

\subsubsection{Principle}

The LDH method measures the oxidation of L-lactate to pyruvate with simultaneous reduction of nicotinamide adenine dinucleotide (NAD). The change in absorbance at $340 \mathrm{~nm}$ due to the appearance of reduced NAD (NADH) is directly proportional to the $\mathrm{LDH}$ activity, since other reactants are present in non-rate limiting quantities and is measured using a bichromatic (340, $383 \mathrm{~nm}$ ) rate technique.

f. Identification and the estimation of the density of Plasmodium parasite in the subjects were carried out as follows: Plasmodium spp was determined in the blood of the control and the test subjects using Giemsha thick blood staining technique described by Cheesbrough [7]. Estimation of parasite number was carried out by multiplying average number of parasites per high power field(100x objective) by 500 within 10 fields $[7,8]$.

\subsubsection{Ethical Consideration}

The proposal was reviewed and approved by the Research and Ethical Committee of General Hospital Kishi-Oyo state- Nigeria before the commencement of the work. This is to protect the interest of patients to ensure that the patients and the community are not harmed in any form by the procedure. Only subjects that volunteered themselves for the study were recruited.

\subsubsection{Statistical Analysis}

The result obtained was subjected to statistical analysis to determine mean, standard deviation, correlation coefficient and ' $\mathrm{t}$ ' test at 0.05 level of significance using online :

Pearson Correlation CoefficientCalculator

http://www.socscistatistics.com/tests/pearson/Default2. aspx

T-Test Calculator for 2 Dependent Means:

www.socscistatistics.com/tests/ttestdependent/

A T-test calculator that compares 2 dependent population means for statistical significance.

\section{Result}

Table 1. Frequencies of Plasmodium infection and its coinfection with HIV, HBV and HCV in the subjects studied

\begin{tabular}{|c|c|c|c|}
\hline & Female & Male & Total \\
\hline Total number screened & $403(56.8 \%)$ & $306(43.2 \%)$ & 709 \\
\hline $\begin{array}{c}\text { Number of plasmodium } \\
\text { infected patients }\end{array}$ & $107(52 \%)$ & $99(48 \%)$ & $206(29.1 \%)$ \\
\hline $\begin{array}{c}\text { Number of plasmodium } \\
\text { infected patients with } \\
\text { coinfection of one or more } \\
\text { of HIV, HCVand HBV }\end{array}$ & $61(53.5 \%)$ & $53(46.5 \%)$ & $114(16.1 \%)$ \\
\hline $\begin{array}{c}\text { Frequency of Plasmodium } \\
\text { infected patients free of } \\
\text { HIV, HBsAg and HCV }\end{array}$ & $58(63.0 \%)$ & $34(37 \%)$ & $92(12.97 \%)$ \\
\hline
\end{tabular}

Table 2. The value of the Falciparum parasite density, Plasma TBA and LDH

\begin{tabular}{|c|c|c|c|c|}
\hline \multicolumn{3}{|c|}{ Parasite density } & \multicolumn{2}{c|}{$\begin{array}{c}\text { Mean and the standard } \\
\text { deviation of plasma TBA and } \\
\text { LDH }\end{array}$} \\
\hline $\begin{array}{c}\text { Range/ } \\
\mu \mathrm{L}\end{array}$ & $\mathrm{N}$ & $\begin{array}{c}\text { Mean and } \\
\text { standard } \\
\text { deviation/ } \mu \mathrm{L}\end{array}$ & $\begin{array}{c}\text { TBA } \\
(\mu \mathrm{mol} / \mathrm{L})\end{array}$ & $\begin{array}{c}\mathrm{LDH} \\
(\mathrm{U} / \mathrm{L})\end{array}$ \\
\hline $50-499$ & $42(45.7 \%)$ & $282 \pm 12.0$ & $10 \pm 1.2$ & $274 \pm 20$ \\
\hline $\begin{array}{c}500- \\
999\end{array}$ & $40(43.5 \%)$ & $853 \pm 31.0$ & $13 \pm 2.6$ & $309 \pm 26.0$ \\
\hline$\geq 1000$ & $10(10.9 \%)$ & $1130 \pm 61.0$ & $16 \pm 2.5$ & $370 \pm 20.0$ \\
\hline
\end{tabular}

\begin{tabular}{|c|c|c|c|c|c|c|}
\hline & $\begin{array}{c}50-499 \\
\text { and } \\
500- \\
999\end{array}$ & $\begin{array}{c}50- \\
499 \\
\text { and } \\
\geq 1000\end{array}$ & $\begin{array}{c}500-999 \\
\text { and } \\
\geq 1000\end{array}$ & $\begin{array}{c}50-499 \\
\text { and } \\
500- \\
999\end{array}$ & $\begin{array}{c}50- \\
499 \\
\text { and } \\
500- \\
999\end{array}$ & $\begin{array}{c}50- \\
499 \\
\text { and } \\
\geq 1000\end{array}$ \\
\hline & \multicolumn{3}{|c|}{ LDH } & \multicolumn{3}{|c|}{ TBA } \\
\hline ' $t$ ' & 10.86 & 9.3 & 13.9 & 6.04 & 9.9 & 10.7 \\
\hline 'p' & 0.0001 & 0.002 & $\begin{array}{c}0.00003 \\
4\end{array}$ & 0.002 & 0.0002 & 0.0001 \\
\hline $\begin{array}{c}\text { Comme } \\
\text { nt }\end{array}$ & $\underset{\mathrm{s}}{\mathrm{p}<0.05}$ & $\underset{\mathrm{s}}{\mathrm{p}<0.05}$ & $\mathrm{p}<0.05^{\mathrm{S}}$ & $\underset{\mathrm{s}}{\mathrm{p}<0.05}$ & $\underset{\mathrm{s}}{\mathrm{p}<0.05}$ & $\underset{\mathrm{s}}{\mathrm{p}<0.05}$ \\
\hline
\end{tabular}

Table 4. Correlative analysis of the values of the Plasmodium parasite density, Plasma TBA and LDH in the patients

\begin{tabular}{|c|c|c|c|c|}
\hline & & $\begin{array}{c}50- \\
499(282 \pm 12 . \\
0) / \mu \mathrm{L}\end{array}$ & $\begin{array}{c}500- \\
999(853 \pm 31 . \\
0) / \mu \mathrm{L}\end{array}$ & $\begin{array}{c}\geq 1000 \\
(1130 \pm 61 . \\
0) \\
/ \mu \mathrm{L}\end{array}$ \\
\hline \multirow{3}{*}{$\begin{array}{c}\text { Values of } \\
\text { the parasite } \\
\text { density/valu } \\
\text { es of plasma } \\
\text { TBA }\end{array}$} & $\mathrm{R}$ & 1 & 1 & 1 \\
\hline & $\mathrm{R}^{2}$ & 1 & 1 & 1 \\
\hline & $\begin{array}{c}\text { Correlati } \\
\text { on }\end{array}$ & $\begin{array}{c}\text { Strong } \\
\text { positive } \\
\text { correlation }\end{array}$ & $\begin{array}{c}\text { Strong } \\
\text { positive } \\
\text { correlation }\end{array}$ & $\begin{array}{c}\text { Strong } \\
\text { positive } \\
\text { correlation }\end{array}$ \\
\hline \multirow{3}{*}{$\begin{array}{l}\text { Values of } \\
\text { the parasite } \\
\text { density/valu } \\
\text { es of LDH }\end{array}$} & $\mathrm{R}$ & 1 & 1 & 1 \\
\hline & $\mathrm{R}^{2}$ & 1 & 1 & 1 \\
\hline & $\begin{array}{c}\text { correlatio } \\
\mathrm{n}\end{array}$ & $\begin{array}{c}\text { Strong } \\
\text { positive } \\
\text { correlation }\end{array}$ & $\begin{array}{c}\text { Strong } \\
\text { positive } \\
\text { correlation }\end{array}$ & $\begin{array}{c}\text { Strong } \\
\text { positive } \\
\text { correlation }\end{array}$ \\
\hline
\end{tabular}

Out of Seven hundred and nine (709) subjects that were screened for Plasmodium infection including female $(n=403)$ and male: $(n=306)$ aged 5 to 68 years it was found that the overall prevalence of Plasmodium infection among the population was 29.1\%(206) (female: 107 (52\%); male: 99 (48\%)) including 12.97\% (92) (Female: 58 (63.0\%) Male: 34 (37\%)) HIV, HBsAg and anti-HCV seronagative patients and $16.1 \%$ (114) (Female: 61 (53.5\%); Male: 53 (46.5\%)) patients infected with at least one of HIV, HBV or HCV (Table $1 \&$ Table 2).

The plasmodium infected subjects were grouped into three based on the parasite density such as: patients with parasite density of 50-499; 500-999 and $\geq 1000$. 
The pattern of parasite density obtained in the rural community studied include $45.7 \%$ had a mean parasite density of $282 \pm 12.0 ; 43.5 \% \quad(853 \pm 31.0)$ and $10.9 \%$ (1130 \pm 61.0$)$. The mean value of each group was correlated with those of LDH and TBA. In all groups there was a strong positive correlation $\left(R=1 ; R^{2}=1\right)$ between the plasma TBA, LDH compared with the plasmodium parasite density. There was also a statistical significant increase in the mean value of LDH and TBA with increase in parasite density with $\mathrm{p}<0.05$ (Table $3 \&$ Table 4).

\section{Discussion, Recommendation}

The overall prevalence of Plasmodium infection was 29.1\% (206) (female: 107 (52\%); male: 99 (48\%)) which was found to be more prevalent in females than the males. This finding is comparable with the report of Ekpo et al [9] among on a total of 527 blood samples collected from settled Fulani pastoralists in Kwara, Oyo and Ogun States respectively that the overall prevalence of malaria infection in the zone was $33.6 \%$. Kwara State had the highest prevalence of $39.0 \%$ while Oyo State had the least prevalence of $29.9 \%$. The mean values of the parasite density obtained indicates plasmodium parasitaemia. Ekpo et al [9] also reported a gender difference in the prevalence of plasmodium infection that female were significantly more infected than their male counterparts which also agrees with the findings of this work..

Out of the $206(29.1 \%)$ subjects infected with plasmodium, 12.97\% (92) (Female: 58 (63.0\%) Male: 34 (37\%)) were HIV, HBs Ag and anti-HCV seronagative patients and 16.1\% (114) (Female: 61 (53.5\%); Male: 53 (46.5\%)) were patients infected with at least one of HIV, $\mathrm{HBV}$ or HCV which is more prevalent in females than males. The coinfection obtained in this study is consistent with the findings of Jain et al., [10] that also reported malaria coinfection with the virus in view in a study conducted to evaluate the seroprevalence of HIV, HBV, HCV, and Syphilis and Malaria among blood donors in a total of 46,224 blood donors during a period from April 2008 to October 2012, at blood bank, S.R.G. Hospital and Medical College Jhalawar - District, Rajasthan State and found that the seropositivity for Human Immunodeficiency Virus (HIV) was 0.034\%, Hepatitis B Virus (HBV) was $1.57 \%$, Hepatitis C Virus (HCV) was 0.04\%, Rapid plasma Reagin method (RPR) for syphilis was $0.019 \%$ and Malaria was $0.017 \%$ respectively. However, the findings of this work was not in agreement with the findings of Allauddin et al., [11] that out of donors who were tested, 3520 were male and 907 female; amongst them, 66 (1.5\%) were positive for HBsAg, and $56(1.3 \%)$ were positive for anti-HCV antibodies. All donors tested negative for HIV and malaria.

The plasmodium infected subjects were grouped into three based on the parasite density such as: patients with parasite density of 50-499; 500-999 and $\geq 1000$.

The mean value of each group was correlated with those of LDH and TBA. In all groups there was a strong positive correlation $(\mathrm{R}=1$; $\mathrm{R} 2=1)$ between the plasma TBA, LDH compared with the plasmodium parasite density. There was also a statistical significant increase in the mean value of $\mathrm{LDH}$ and TBA with increase in parasite density with $\mathrm{p}<0.05$. These indicate; that the higher the plasmodium parasite density the higher the TBA and LDH plasma level and the higher the parasite density the higher the mortality [7]. Therefore the measurement of these two parameters in the plasma of the plasmodium infected patients could be an indication of the degree of severity of the infection and liver dysfunction because LDH is widely distributed throughout the body; highest concentrations are found in the liver, heart and skeletal muscle. $\mathrm{LDH}$ is a general marker of tissue damage and is often used to determine the root cause and location of damage. Plasmodium infection is also associated with haemolysis/tissue destruction. The larger the plasmodium density the higher the destruction and the more the effect on the liver because liver is also involved at the preerythrocytic stage of the life cycle of Plasmodium which may affect its normal metabolic activities may also account for these findings [2].

Furthermore, the measurement of Total bile acids (TBA) in serum is a sensitive indicator of liver function. Bile acid measurement may detect some forms of liver disease earlier than standard liver tests because bile acids levels correspond to liver function, rather than liver damage. Bile acid measurement is considered to be a superior indicator of liver disease there could be liver dysfunction / hepatopathy in malaria which may not be conveniently associated with the hepatotocxic effect of raw liquid extract of Morinda lucida. This is because there was no significant difference in the result obtained in the plasmodium infected patients when the plasma ALT and TBA levels before the administration of the extract were compared with the result obtained in the patients after treatment which may rule out hepatotoxicity as this also agrees with the findings of Oduola et al., [12] that evaluated hepatotoxicity and nephrotoxicity in Wistar albino rats exposed to Morinda lucida leaf extract and found that ingestion of Morinda lucida leaf extract has no toxic effect on liver and kidney functions.

The findings of this study could also be generally be attributed to the reports of Uzuegbu and Emeka [13] and Bhalla et al., [14] that there is evidence of liver dysfunction among the malaria infected patients.

\section{Conclusion}

This work showed an overall prevalence of 29.1\% (206) plasmodium infection including $16.1 \%$ (114) of the patients co-infected with at least one of HIV, HBV or HCV. The plasma level of LDH and TBA was also found to be positively correlated and directly proportional to the parasite density. The pattern of parasite density obtained in the rural community studied include $45.7 \%$ had a mean parasite density of $282 \pm 12.0 ; 43.5 \% \quad(853 \pm 31.0)$ and $10.9 \%(1130 \pm 61.0)$

\section{Recommendation}

Periodical evaluation of the prevalence of plasmodium infection and routine quantification of the parasite density, plasma LDH and TBA in plasmodium infected patients will provide useful directions for effective control and management of malaria. 


\section{References}

[1] Brian M. Greenwood, David A. Fidock, Dennis E. Kyle, Stefan H.I. Kappe, Pedro L. Alonso, Frank H. Collins, Patrick E. Duffy. Malaria: progress, perils, and prospects for eradication. J. Clin. Invest.; 118: 1266-1276. 2008. Full Text at http://www.jci.org/articles/view/33996/files/pdf

[2] Azuma M, Shi M, Danenberg KD, Gardner H, Barrett C, Jacques $\mathrm{CJ}$ et al. "Serum lactate dehydrogenase levels and glycolysis significantly correlate with tumor VEGFA and VEGFR expression in metastatic CRC patients.". Pharmacogenomics: 8 (12): 1705-13. 2007.

[3] Russell DW. "The enzymes, regulation, and genetics of bile acid synthesis". Annu. Rev. Biochem.; 72: 13774, 2003.

[4] Shima T1, Tada H, Morimoto M, Nakagawa Y, Obata H, Sasaki T, Park H, Nakajo S, Nakashima T, Okanoue T, Kashima K.Serum total bile acid level as a sensitive indicator of hepatic histological improvement in chronic hepatitis $\mathrm{C}$ patients responding to interferon treatment. J Gastroenterol Hepatol. Mar; 15 (3): 294-9, 2000.

[5] Chiang JY. "Bile acids: regulation of synthesis". J. Lipid Res.: 50 (10): 1955-66. 2009. PMC 2739756. PMID 19346330.

[6] Hofmann AF. "The continuing importance of bile acids in liver and intestinal disease". Arch. Intern. Med.: 159 (22): 2647-58, 1999.

[7] Greenwood BM, Armstrong JRM. Comparison of two simple methods for determining malaria parasite density. Transactions of the Royal Society of Tropical Medicine and Hygiene.; 85: 186188, 1991.

[8] Cheesbrough, Monica. District Laboratory practice in tropical Countries part1. 2002: Cambridge low-price edition. Cambridge University Press.

[9] UF Ekpo, AM Omotayo, MA Dipeolu. Sendentarization and the prevalence of malaria and anaemia among settled Fulani pastoralists in south-western Nigeria. Nigerian Journal of Parasitology Vol. 29 (2): pp. 125-130, 2008.

[10] Jain. C, N.C. Mogra, Jhaman Mehta, Rishi Diwan, Gaurav Dalela.Comparison Of Seropositivity Of Hiv, Hbv, Hcv And Syphilis And Malaria In Replacement And Voluntary Blood Donors In Western India. IJCRR.; 5 (3): 43-46, 2013.

[11] Allauddin Niazi, Mohammad Tahir, Khalid Farooq Danish, Muhammad Abid, Shazia. Low Seroprevalence of HBV, HCV, HIV and Malaria in Voluntary Non Remunerated Blood Donors. Ann. Pak. Inst. Med. Sci.; 4 (3): 162-164, 2008.

[12] Oduola. T, Bello. I and Avwioro. G. Hepatotoxicity and nephrotoxicity evaluation in Wistar albino rats exposed to Morinda lucida leaf extract. N Am J Med Sci. 2 (5): 230-233. 2010

[13] Uzuegbu U.E. and Emeka C.B. Changes in Liver Function Biomarkers among Malaria Infected Patients in Ikeja Lagos State, Nigeria. Current Research Journal of Biological Sciences: 3 (3): 172-174, 2010. 2011 ISSN: 2041-0778.

[14] Bhalla A, Suri V, Singh V. "Malarial hepatopathy". Journal of Postgraduate Medicine: 52 (4): 315-20. 2006 PMID 17102560. 